\title{
COMODULES AND CONTRAMODULES
}

\author{
ROBERT WISBAUER \\ Department of Mathematics, Heinrich Heine University, 40225 Düsseldorf, Germany \\ e-mail:wisbauer@math.uni-duesseldorf.de
}

\begin{abstract}
Algebras $A$ and coalgebras $C$ over a commutative ring $R$ are defined by properties of the (endo)functors $A \otimes_{R}-$ and $C \otimes_{R}-$ on the category of $R$-modules ${ }_{R} \mathbb{M}$. Generalising these notions, monads and comonads were introduced on arbitrary categories, and it turned out that some of their basic relations do not depend on the specific properties of the tensor product. In particular, the adjoint of any comonad is a monad (and vice versa), and hence, for any coalgebra $C, \operatorname{Hom}_{R}(C,-)$, the right adjoint of $C \otimes_{R}-$, is a monad on ${ }_{R} \mathbb{M}$. The modules for the monad $\operatorname{Hom}_{R}(C,-)$ were called contramodules by Eilenberg-Moore and the purpose of this talk is to outline the related constructions and explain the relationship between $C$-comodules and $C$ contramodules. The results presented grew out from cooperation with G. Böhm, T. Brzeziński and B. Mesablishvili.
\end{abstract}

2010 Mathematics Subject Classification. 16T15, 18C20.

1. Preliminaries. In this section we recall some formalisms for module categories and their transfer to arbitrary categories. Throughout $R$ will denote a commutative ring with unit and $R_{R} \mathbb{M}$ the category of (left) $R$-modules. We recall (once again) the basic definitions explicitly since they provide the prototype of the constructions to follow.

For any $R$-modules $N, X, N \otimes_{R} X$ is again an $R$-module and thus we have a functor (endofunctor)

$$
\begin{aligned}
& N \otimes_{R}-:{ }_{R} \mathbb{M} \rightarrow{ }_{R} \mathbb{M}, \quad X \mapsto N \otimes_{R} X, \\
& f: X \rightarrow Y \mapsto I_{N} \otimes f: N \otimes_{R} X \rightarrow N \otimes_{R} Y .
\end{aligned}
$$

1.1. Algebras. An $R$-module $A$ is called an $R$-algebra provided there is an associative $R$-bilinear form $\tilde{m}: A \times A \rightarrow A,(a, b) \mapsto a b$, and there exists a unit element $1_{A}$. Forming the tensor product, the map $\tilde{m}$ can be replaced by an $R$ linear map $m: A \otimes_{R} A \rightarrow A, a \otimes b \mapsto a b$, and the unit leads to an $R$-linear map $e: R \rightarrow A, r \mapsto r \cdot 1_{A}$. Thus, an $R$-algebra $A$ is characterised by two morphisms in ${ }_{R} \mathrm{M}$ - multiplication and unit

$$
m: A \otimes_{R} A \rightarrow A, \quad e: R \rightarrow A,
$$

and associativity and unitality are expressed by the commutative diagrams
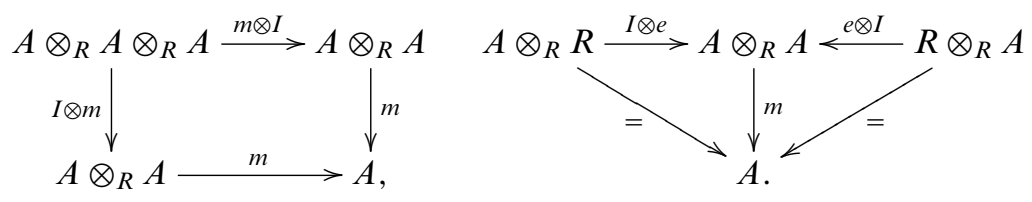
1.2. Category of $A$-modules. Let $(A, m, e)$ be an $R$-algebra. An $R$-module $M$ is said to be an $A$-module if there is an $R$-linear map

$$
\rho_{M}: A \otimes_{R} M \rightarrow M, \quad a \otimes m \mapsto a m,
$$

inducing commutative diagrams

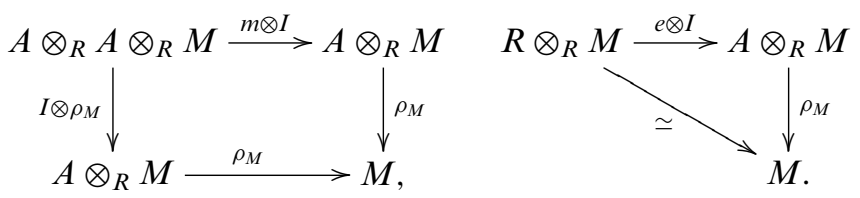

A-module morphisms $f: M \rightarrow N$ are $R$-linear maps inducing commutative diagrams

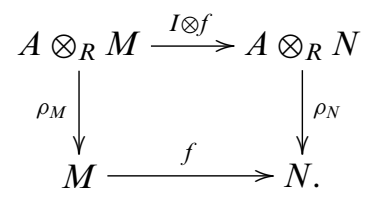

The category of left $A$-modules is denoted by ${ }_{A} \mathbb{M}$. For any $R$-module $X, A \otimes_{R} X$ is an $A$-module with multiplication induced by the algebra product $m$. This yields a functor (denoted by the same symbol as the endofunctor)

$$
A \otimes_{R}-:{ }_{R} \mathbb{M} \rightarrow{ }_{A} \mathbb{M}, \quad X \mapsto\left(A \otimes_{R} X, m \otimes I_{X}\right),
$$

which is left adjoint to the forgetful functor $U_{A}:{ }_{A} \mathbb{M} \rightarrow{ }_{R} \mathbb{M}$, that is, there is a functorial isomorphism

$$
\operatorname{Hom}_{A}\left(A \otimes_{R} M, X\right) \rightarrow \operatorname{Hom}_{R}(M, X), \quad f \mapsto f\left(1_{A} \otimes-\right)
$$

1.3. Coalgebras. An $R$-module $C$ is called an $R$-coalgebra provided there are $R$ linear maps - comultiplication and counit

$$
\Delta: C \rightarrow C \otimes_{R} C, \quad \varepsilon: C \rightarrow R
$$

with coassociativity and counitality conditions expressed by inverting the arrows in the diagrams in 1.1.

1.4. Category of comodules. Let $(C, \Delta, \varepsilon)$ be an $R$-coalgebra. A left $C$-comodule is an $R$-module $M$ with an $R$-linear map

$$
\varrho^{M}: M \rightarrow C \otimes_{R} M
$$

with compatibility conditions obtained from those in 1.2 by reversing arrows. 
$C$-comodule morphisms $f: M \rightarrow N$ are $R$-linear maps inducing commutative diagrams

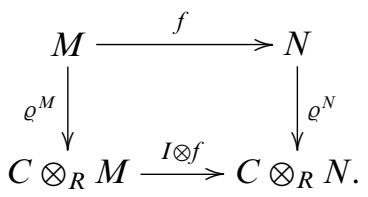

The category of left $C$-comodules is denoted by ${ }^{C} \mathbb{M}$. For any $R$-module $X, C \otimes_{R} X$ is a left $C$-comodule with coaction induced by $\Delta$. This yields a functor

$$
C \otimes_{R}-:{ }_{R} \mathbb{M} \rightarrow{ }^{C} \mathbb{M}, \quad X \mapsto\left(C \otimes_{R} X, \Delta_{X}\right),
$$

which is right adjoint to the forgetful functor $U^{C}:{ }^{C} \mathbb{M} \rightarrow{ }_{R} \mathbb{M}$, that is, there is an isomorphism

$$
\operatorname{Hom}^{C}\left(M, C \otimes_{R} X\right) \rightarrow \operatorname{Hom}_{R}(M, X), \quad f \mapsto\left(\varepsilon \otimes I_{X}\right) \circ f .
$$

Transferring the construction just revisited to arbitrary categories leads to the following notions. By $\mathbb{A}$ we denote any category.

First we express the properties of an algebra in functor terms. Note that $A \otimes_{R} A$ may be seen as composition of the functor $A \otimes_{R}-$ with $A \otimes_{R}-$.

1.5. Monad on $\mathbb{A}$. An endofunctor $F: \mathbb{A} \rightarrow \mathbb{A}$ is called a monad on $\mathbb{A}$ provided there are natural transformations

$$
m: F F \rightarrow F, \quad e: I_{\AA} \rightarrow F,
$$

satisfying associativity and unitality conditions (as for algebras, see 1.1).

1.6. $F$-modules. Let $(F, m, e)$ be a monad on $\mathbb{A}$. An object $A$ in $\mathbb{A}$ is an $F$-module provided there is a morphism

$$
\varrho_{A}: F(A) \rightarrow A \text { in } \mathbb{A},
$$

satisfying associativity and unitality conditions (as for $A$-modules, see 1.2).

An $F$-module morphism $f: A \rightarrow B$ between $F$-modules is a morphism in $\mathbb{A}$ subject to commutativity of the diagram

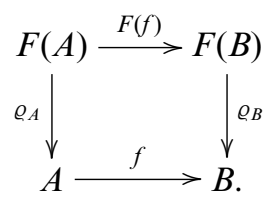

The category of $F$-modules, also called the Eilenberg-Moore category of $F$, is denoted by $\mathbb{A}_{F}$. For any object $X$ in $\mathbb{A}, F(X)$ is an $F$-module with multiplication induced by $m$. This leads to a functor

$$
\phi_{F}: \mathbb{A} \rightarrow \mathbb{A}_{F}, \quad X \mapsto\left(F(X), m_{X}\right),
$$


which is left adjoint to the forgetful functor $U_{F}: \mathbb{A}_{F} \rightarrow \mathbb{A}$, by the functorial isomorphism, for $X \in \mathbb{A}, N \in \mathbb{A}_{F}$,

$$
\operatorname{Mor}_{F}(F(X), N) \rightarrow \operatorname{Mor}_{\AA}(X, N), \quad h \mapsto h \circ e_{X} .
$$

Similar to algebras, the notion of a coalgebra also transfers to general categories. Again $\mathbb{A}$ denotes any category.

1.7. Comonad on $\mathbb{A}$. An endofunctor $G: \mathbb{A} \rightarrow \mathbb{A}$ is called a comonad if there are natural transformations

$$
\delta: G \rightarrow G G, \quad \varepsilon: G \rightarrow I_{\AA},
$$

satisfying coassociativity and counitality conditions (as in 1.3).

1.8. $G$-comodules. Let $(G, \delta, \varepsilon)$ be a comonad on $\mathbb{A}$. An object $A$ in $\mathbb{A}$ is a $G$ comodule provided there exists a morphism

$$
\varrho^{A}: A \rightarrow G(A) \text { in } \mathbb{A},
$$

subject to coassociativity and counitality conditions (as in 1.4).

$G$-comodule morphisms $g: A \rightarrow B$ between $G$-comodules are morphisms in $\mathbb{A}$ inducing commutative diagrams

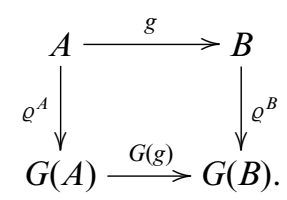

The category of $G$-comodules, also called the Eilenberg-Moore category of $G$, is denoted by $\mathbb{A}^{G}$. For any $X$ in $\mathbb{A}, G(X)$ is a $G$-comodule with coaction induced by $\delta$. This yields a functor

$$
\phi^{G}: \mathbb{A} \rightarrow \mathbb{A}^{G}, X \mapsto\left(G(X), \delta_{X}\right),
$$

which is right adjoint to the forgetful functor $U^{G}: \mathbb{A}^{G} \rightarrow \mathbb{A}$ by the functorial isomorphism

$$
\operatorname{Mor}^{G}(M, G(X)) \rightarrow \operatorname{Mor}_{\mathbb{A}}(M, X), \quad f \mapsto \varepsilon_{X} \circ f .
$$

Note that for modules of a monad (1.6) as well as for comodules of a comonad (1.7), we have obtained the same basic relations as for $A$-modules (1.2) and for $C$ comodules (1.4), respectively. While the latter are derived from the tensor functor (hence a functor with specific properties), we did not impose any condition on the monads or comonads. Bringing these abstract notions back to module categories, the question arises if there are other endofunctors on ${ }_{R} \mathbb{M}$ which allow for a monad or comonad structure. Some answers to this will be given in the next section.

2. Adjoint endofunctors. We first consider the general case. So let $\mathbb{A}$ be any category. Recall that an endofunctor $F: \mathbb{A} \rightarrow \mathbb{A}$ is left adjoint to an endofunctor 
$G: \mathbb{A} \rightarrow \mathbb{A}$ provided there is a functorial isomorphism

$$
\varphi_{X, Y}: \operatorname{Mor}_{\AA}(F(X), Y) \longrightarrow \operatorname{Mor}_{\AA}(X, G(Y)) \text {. }
$$

2.1. Adjoints of monads. Let $(F, G)$ be an adjoint pair of endofunctors on $\mathbb{A}$. Assume $F$ to be a monad with $m: F F \rightarrow F, e: I_{\mathbb{A}} \rightarrow F$. Then we obtain the diagram

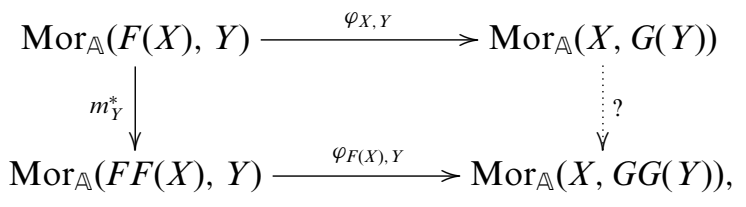

where $m^{*}=\operatorname{Mor}_{\mathbb{A}}(m,-)$ and the question mark can be replaced by the natural transformation

$$
\varphi_{F(X), Y} \circ m_{Y}^{*} \circ \varphi_{X, Y}^{-1} .
$$

Together with a corresponding diagram for the unit we conclude by the Yoneda lemma that there is a comonad structure on $G$ :

$$
\delta: G \rightarrow G G, \quad \varepsilon: G \rightarrow I_{\mathbb{A}} .
$$

It may come as a surprise that, in the situation considered in 2.1 , the category of $F$-modules is isomorphic to the category of $G$-comodules.

2.2. Modules and comodules for an adjoint pair. Let $(F, G)$ be an adjoint pair of functors with unit $\eta: I_{\mathbb{A}} \rightarrow G F$ and counit $\varepsilon: F G \rightarrow I_{\AA}$. Let $F$ be a monad and consider $G$ with the induced comonad structure. Then the (Eilenberg-Moore) categories $\mathbb{A}_{F}$ and $\mathbb{A}^{G}$ are isomorphic to each other.

Proof. The isomorphism is given by a functor leaving objects and morphisms unchanged and turning $F$-module structure maps to $G$-comodule structure maps and vice versa. An $F$-module $\varrho_{A}: F(A) \rightarrow A$ induces a morphism

$$
A \stackrel{\eta A}{\longrightarrow} G F(A) \stackrel{G \varrho_{A}}{\longrightarrow} G(A),
$$

making $A$ a $G$-comodule. Similarly, a comodule $\varrho^{A}: A \rightarrow G(A)$ induces

$$
F(A) \stackrel{F Q^{A}}{\longrightarrow} F G(A) \stackrel{\varepsilon_{A}}{\longrightarrow} A
$$

defining an $F$-module structure on $A$.

It remains to show that module morphisms are also comodule morphisms. An $F$-module morphism $f: A^{\prime} \rightarrow A$ yields a commutative diagram

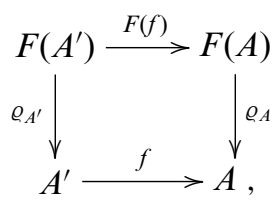


from which we obtain the commutative diagram

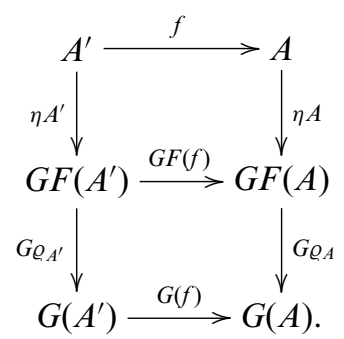

Commutativity of the outer rectangle shows that $f$ is also a $G$-comodule morphism. Similarly one proves that $G$-comodule morphisms are also $F$-module morphisms.

2.3. Adjoints of comonads. Let $(F, G)$ be an adjoint pair of endofunctors on $\mathbb{A}$. Now assume $F$ to be a comonad. Then-with similar arguments as in 2.1 - one can see that $G$ has a monad structure.

Related to such a pair we have the categories $\mathbb{A}^{F}$ and $\mathbb{A}_{G}$. Unlike the monadcomonad case considered in 2.2 , here we do not get an equivalence between $\mathbb{A}^{F}$ and $\mathbb{A}_{G}$. There is, however, an equivalence between certain subcategories (Kleisli subcategories, see [1, Section 2]). We will come back to this problem for ${ }_{R} \mathbb{M}$ (in 3.9).

Having the general setting developed above, one may ask about the meaning of this in the category of $R$-modules. For this, recall the following.

2.4. Adjoint endofunctors on ${ }_{R} \mathbb{M}$. For any $R$-module $M$, the endofunctors $M \otimes_{R}-$ and $\operatorname{Hom}_{R}(M,-)$ form an adjoint pair by the isomorphism

$$
\psi_{X, Y}^{M}: \operatorname{Hom}_{R}\left(M \otimes_{R} X, Y\right) \rightarrow \operatorname{Hom}_{R}\left(X, \operatorname{Hom}_{R}(M, Y)\right), \quad \gamma \mapsto[x \mapsto \gamma(-\otimes x)],
$$

with unit $v: I \rightarrow \operatorname{Hom}_{R}\left(M, M \otimes_{R}-\right)$ and counit $\varepsilon: M \otimes_{R} \operatorname{Hom}(M,-) \rightarrow I$.

First we apply 2.1 and 2.2 to algebras.

Proposition 2.5. For an $R$-module $A$ the following are equivalent:

(a) $A$ is an R-algebra.

(b) $A \otimes_{R}-:{ }_{R} \mathbb{M} \rightarrow{ }_{R} \mathbb{M}$ is a monad.

(c) $\operatorname{Hom}_{R}(A,-):{ }_{R} \mathbb{M} \rightarrow{ }_{R} \mathbb{M}$ is a comonad.

In this case the categories ${ }_{A} \mathbb{M}=\mathbb{M}_{A \otimes_{R^{-}}}$and $\mathbb{M}^{\operatorname{Hom}(A,-)}$ are isomorphic.

Proof. The assertions are special cases of 2.1 and 2.2. The algebra structure on $A, m: A \otimes_{R} A \rightarrow A$ and $e: R \rightarrow A$ correspond to the comonad structure on $\operatorname{Hom}_{R}(A,-)$ :

$$
\begin{aligned}
\operatorname{Hom}_{R}(A,-) \stackrel{m^{*}}{\longrightarrow} & \operatorname{Hom}_{R}\left(A \otimes_{R} A,-\right) \stackrel{\psi_{A,-}^{A}}{\longrightarrow} \operatorname{Hom}_{R}\left(A, \operatorname{Hom}_{R}(A,-)\right), \\
& \operatorname{Hom}_{R}(A,-) \stackrel{e^{*}}{\longrightarrow} \operatorname{Hom}_{R}(R,-),
\end{aligned}
$$

where $m^{*}=\operatorname{Hom}_{R}(m,-)$ and $e^{*}=\operatorname{Hom}_{R}(e,-)$. 
The $A$-module structure $\rho_{N}: A \otimes_{R} N \rightarrow N$ induces a $\operatorname{Hom}_{R}(A,-)$-comodule structure on $N$ :

$$
\widehat{\rho_{N}}: N \stackrel{v_{N}}{\longrightarrow} \operatorname{Hom}_{R}\left(A, A \otimes_{R} N\right) \stackrel{\operatorname{Hom}\left(A, \rho_{N}\right)}{\longrightarrow} \operatorname{Hom}_{R}(A, N)
$$

On the other hand, a comodule structure $\rho^{N}: N \rightarrow \operatorname{Hom}_{R}(A, N)$ induces an $A$-module structure on $N$ :

$$
\widetilde{\rho^{N}}: A \otimes_{R} N \stackrel{I \otimes \rho^{N}}{\longrightarrow} A \otimes_{R} \operatorname{Hom}_{R}(A, N) \stackrel{\varepsilon_{N}}{\longrightarrow} N
$$

The equivalence between the module and comodule categories is given by

$$
\mathbb{M}_{A \otimes_{R^{-}}} \rightarrow \mathbb{M}^{\operatorname{Hom}(A,-)}, \quad\left(M, \rho_{N}\right) \mapsto\left(M, \widehat{\rho_{N}}\right),
$$

keeping the morphisms unchanged.

2.6. Value at $R$. Since $R$ is a generator in ${ }_{R} \mathbb{M}$ and $A \otimes_{R}-$ preserves direct sums and epimorphisms, the functor $A \otimes_{R}-:{ }_{R} \mathbb{M} \rightarrow{ }_{R} \mathbb{M}$ is fully determined by the value at $R$, that is, by $A \otimes_{R} R \simeq A$. Similarly, a natural transformation $\varphi: A \otimes_{R}-\rightarrow B \otimes_{R}-$ between tensor functors is of the form $\varphi_{R} \otimes_{R}-$, where $\varphi_{R}: A \rightarrow B$ is an $R$-linear map.

In general, $\operatorname{Hom}_{R}(A,-)$ is not determined by $A^{*}=\operatorname{Hom}_{R}(A, R)$, unless it preserves direct sums and epimorphisms, that is, unless $A$ is a finitely generated and projective $R$-module. However, $\operatorname{Hom}_{R}(A,-)$ is determined by $\operatorname{Hom}_{R}(A, Q)$ for any cogenerator $Q \in R_{R} \mathbb{M}$ since it is left exact and preserves direct products. For a natural transformation $\psi: \operatorname{Hom}_{R}(A,-) \rightarrow \operatorname{Hom}_{R}(B,-)$ between Hom functors, it follows by the Yoneda lemma that $\psi=\operatorname{Hom}_{R}\left(\psi_{R},-\right)$, where $\psi_{R}: B \rightarrow A$ is an $R$-linear map.

3. Comodules and contramodules. In this section, let $(C, \Delta, \varepsilon)$ be an $R$-coalgebra (see 1.3).

The category ${ }^{C} \mathbb{M}$ of left $C$-comodules is well investigated. Besides the basic facts mentioned in 1.4, there are more relations which partly result from special properties of the functor $C \otimes_{R}-$ as being right exact and preserving coproducts (see [2]).

\subsection{The category ${ }^{C} \mathbb{M}$.}

(1) ${ }^{C} \mathbb{M}$ is an additive category with coproducts and cokernels.

(2) For any generator $P \in{ }_{R} \mathbb{M}, C \otimes_{R} P$ is a subgenerator in ${ }^{C} \mathbb{M}$, in particular, $C$ is a subgenerator in ${ }^{C} \mathbb{M}$.

(3) For any monomorphism $f: X \rightarrow Y$ in ${ }_{R}^{\mathbb{M}, f} f \otimes_{R} I_{C}: C \otimes_{R} X \rightarrow C \otimes_{R} Y$ is a monomorphism in ${ }^{C}$ M.

(4) For any family $X_{\lambda}$ of $R$-modules, $C \otimes_{R} \prod_{\Lambda} X_{\lambda}$ is the product of the $C$ comodules $C \otimes_{R} X_{\lambda}$.

The $R$-module structure of $C$ has a strong influence on the properties of ${ }^{C} \mathbb{M}$ $[2,3.14]$.

Proposition 3.2. For an R-coalgebra $C$, the following are equivalent:

(a) $C$ is flat as an $R$-module.

(b) Every monomorphism in ${ }^{C} \mathbb{M}$ is injective. 
(c) The forgetful functor ${ }^{C} \mathbb{M} \rightarrow{ }_{R} \mathbb{M}$ respects monomorphisms.

In this case, ${ }^{C} \mathbb{M}$ is an abelian category and

(i) for any cogenerator $Q \in{ }_{R} \mathbb{M}, C \otimes_{R} Q$ is a cogenerator in ${ }^{C} \mathbb{M}$;

(ii) for any injective $X \in{ }_{R} \mathbb{M}, C \otimes_{R} X$ is injective in ${ }^{C} \mathbb{M}$.

In view of the adjointness of the endofunctors $C \otimes_{R}-$ and $\operatorname{Hom}_{R}(C,-)$, the latter has a monad structure by 2.3 .

Proposition 3.3. For an $R$-module $C$, the following are equivalent:

(a) $C$ is an R-coalgebra.

(b) $C \otimes_{R}-:{ }_{R} \mathbb{M} \rightarrow{ }_{R} \mathbb{M}$ is a comonad.

(c) $\operatorname{Hom}_{R}(C,-):{ }_{R} \mathbb{M} \rightarrow{ }_{R} \mathbb{M}$ is a monad.

Hereby the coalgebra structure maps $\Delta: C \rightarrow C \otimes_{R} C, \varepsilon: C \rightarrow R$, correspond to the monad structure

$$
\begin{gathered}
\operatorname{Hom}_{R}\left(C, \operatorname{Hom}_{R}(C,-)\right) \stackrel{\simeq}{\longrightarrow} \operatorname{Hom}_{R}\left(C \otimes_{R} C,-\right) \stackrel{\Delta^{*}}{\longrightarrow} \operatorname{Hom}_{R}(C,-), \\
\operatorname{Hom}_{R}(R,-) \stackrel{\varepsilon^{*}}{\longrightarrow} \operatorname{Hom}_{R}(C,-),
\end{gathered}
$$

where $\Delta^{*}=\operatorname{Hom}_{R}(\Delta,-), \varepsilon^{*}=\operatorname{Hom}_{R}(\varepsilon,-)$, and the isomorphism is from 2.4.

Henceforth we will write $[C,-]=\operatorname{Hom}_{R}(C,-)$ for short. By applying 1.6 we have the definition of $[C,-]$-modules and the category $\mathbb{M}_{[C,-]}$ with the (free) functor

$$
\phi_{[C,-]}:{ }_{R} \mathbb{M} \rightarrow \mathbb{M}_{[C,-]}
$$

being left adjoint to the forgetful functor $U_{[C,-]}: \mathbb{M}_{[C,-]} \rightarrow{ }_{R} \mathbb{M}$. Following [4], the $[C,-]$-modules are also called $C$-contramodules.

By left exactness of the functor $[C,-]$ we obtain (with similar arguments as in the comodule case) special properties of $[C,-]$-modules.

3.4. The category $\mathbb{M}_{[C,-]}$. Let $C$ be an $R$-coalgebra.

(1) $\mathbb{M}_{[C,-]}$ is an additive category with products and kernels.

(2) For any $M \in \mathbb{M}_{[C,-]}, \operatorname{Hom}_{[C,-]}([C, R], M) \simeq M$.

(3) For any epimorphism $h: X \rightarrow Y$ in ${ }_{R}^{\mathbb{M},}[C, h]:[C, X] \rightarrow[C, Y]$ is an epimorphism (not necessarily surjective) in $\mathbb{M}_{[C,-]}$.

(4) For any family $X_{\lambda}$ of $R$-modules, $\left[C, \bigoplus_{\Lambda} X_{\lambda}\right]$ is the coproduct of the $[C,-]$ modules $\left[C, X_{\lambda}\right]$.

For $C$-comodules, it is not always clear if the kernel of a $C$-comodule morphism is a subcomodule. Here we need some condition to make the cokernel of a $[C,-]$-module morphism again a $[C,-]$-module.

Proposition 3.5. Let $C$ be an R-algebra. If $C_{R}$ is projective, then for any $[C,-]-$ submodule $K \subset M$, the $R$-module $M / K$ is a $[C,-]$-module. 
Proof. By assumption we have the commutative diagram with exact rows

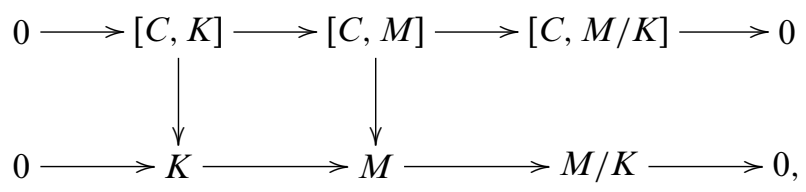

which can be completed commutatively by an $R$-morphism $[C, M / K] \rightarrow M / K$. It is routine to check that this provides $M / K$ with a $[C,-]$-module structure.

As observed above, in $\mathbb{M}_{[C,-]}$ epimorphisms need not be surjective maps. This is the case under the following conditions.

Proposition 3.6. For an $R$-coalgebra $C$, the following are equivalent:

(a) $C_{R}$ is projective.

(b) Every epimorphism in $\mathbb{M}_{[C,-]}$ is surjective.

(c) The forgetful functor $\mathbb{M}_{[C,-]} \rightarrow \mathbb{M}_{R}$ respects epimorphisms.

In this case, $\mathbb{M}_{[C,-]}$ is an abelian category and

(i) for any generator $P \in{ }_{R} \mathbb{M},[C, P]$ is a generator in $\mathbb{M}_{[C,-]}$;

(ii) for any projective $Y \in{ }_{R} \mathbb{M},[C, Y]$ is projective in $\mathbb{M}_{[C,-]}$.

Proof. (b) $\Rightarrow$ (a) For any epimorphism $f: K \rightarrow L$ in ${ }_{R}^{\mathbb{M},} \operatorname{Hom}(C, f)$ : $\operatorname{Hom}_{R}(C, K) \rightarrow \operatorname{Hom}_{R}(C, K)$ is an epimorphism in $\mathbb{M}_{[C,-]}$ and hence surjective by (b). This means that $C_{R}$ is projective.

(c) $\Rightarrow$ (a) is shown with a similar argument.

(a) $\Rightarrow$ (c) Assume $C_{R}$ to be projective and consider an epimorphism $f: M \rightarrow N$ in $\mathbb{M}_{[C,-]}$. Then the cokernel $h: N \rightarrow N / f(M)$ is a morphims in $\mathbb{M}_{[C,-]}$ and $0 f=h f=0$. Since $f$ is an epimorphism, this implies $N=f(M)$.

Recall that for any $R$-coalgebra $C$, the dual space $C^{*}=\operatorname{Hom}_{R}(C, R)$ has a ring structure by the convolution product for $f, g \in C^{*}, f * g=(f \otimes g) \circ \Delta$. The relation between $C$-comodules and modules over the dual ring of $C$ is well studied (e.g. [2, Section 19]). Now it follows from the general observations in 2.1 and 2.3 that a coalgebra $C$ gives rise to two comonads and two monads on ${ }_{R} \mathbb{M}$ :

$$
C \otimes_{R}-\text { and } \operatorname{Hom}_{R}\left(C^{*},-\right), \quad C^{*} \otimes_{R}-\text { and } \operatorname{Hom}_{R}(C,-) .
$$

Relations between these are given by morphisms which are well known in module theory-but usually not viewed under this aspect.

3.7. The comonads $C \otimes_{R}-$ and $\left[C^{*},-\right]$. The comonad morphism

$$
\alpha: C \otimes_{R}-\rightarrow \operatorname{Hom}_{R}\left(C^{*},-\right), \quad c \otimes-\mapsto[f \mapsto f(c)-]
$$

yields a faithful functor

$$
\begin{aligned}
G_{\alpha}: \quad C_{\mathbb{M}} & \longrightarrow \mathbb{M}^{\left[C^{*},-\right]} \simeq \mathbb{M}_{C^{*}}, \\
N \stackrel{\varrho^{N}}{\longrightarrow} C \otimes_{R} N & \longrightarrow N \stackrel{\varrho^{N}}{\longrightarrow} C \otimes_{R} N \stackrel{\alpha_{N}}{\longrightarrow} \operatorname{Hom}_{R}\left(C^{*}, N\right),
\end{aligned}
$$


and the following are equivalent:

(a) $\alpha_{N}$ is injective for each $N \in{ }_{R} \mathbb{M}$.

(b) $G_{\alpha}$ is a full functor.

(c) $C$ is a locally projective left $R$-module.

If these conditions are satisfied, ${ }^{C} \mathbb{M}$ is equal to $\sigma\left[C_{C^{*}}\right]$, the full subcategory of $\mathbb{M}_{C^{*}}$ subgenerated by $C$.

Similar to $3.7, C$-contramodules can be related to $C^{*}$-modules.

3.8. The monads $[C,-]$ and $-\otimes_{R} C^{*}$. The monad morphism

$$
\beta:-\otimes_{R} C^{*} \rightarrow \operatorname{Hom}_{R}(C,-), \quad-\otimes f \mapsto[c \mapsto f(c)-]
$$

yields a faithful functor

$$
\begin{gathered}
F_{\beta}: \quad \stackrel{\mathbb{M}_{[C,-]}}{\longrightarrow} \mathbb{M}_{C^{*}}, \\
\operatorname{Hom}_{R}(C, M) \stackrel{\varrho_{M}}{\longrightarrow} M \longmapsto M \otimes_{R} C^{*} \stackrel{\beta_{M}}{\longrightarrow} \operatorname{Hom}_{R}(C, M) \stackrel{\varrho_{M}}{\longrightarrow} M,
\end{gathered}
$$

and the following are equivalent:

(a) $\beta$ is surjective for all $M \in{ }_{R} \mathbb{M}$.

(b) $F_{\beta}$ is an isomorphism.

(c) $C$ is a finitely generated and projective $R$-module.

In general, $C$ is not a $[C,-]$-module and $[C, R]$ is not a $C$-comodule. In fact, $[C, R] \in{ }^{C} \mathbb{M}$ holds provided $C$ is finitely generated and projective as an $R$-module.

We now consider the relationship between the categories of $C$-comodules and $C$-contramodules. As indicated in 2.3, the two categories need not be equivalent. This becomes evident from their properties listed in 3.4 and 3.1, respectively. However, they are related by an adjoint pair of functors.

3.9. Correspondence of categories. Let $C$ be an $R$-coalgebra. Then

$$
\operatorname{Hom}^{C}(C,-):{ }^{C} \mathbb{M} \rightarrow \mathbb{M}_{[C,-]}, \quad M \mapsto \operatorname{Hom}^{C}(C, M)
$$

is a functor which has a left adjoint given by the contratensor product defined for $(N, \rho) \in{ }^{C} \mathbb{M}$ and $(M, \alpha) \in \mathbb{M}_{[C,-]}$ as the coequaliser

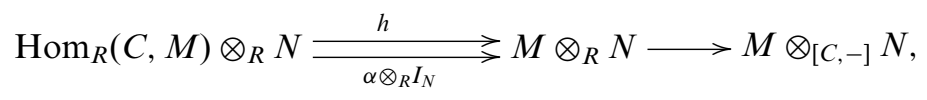

for the map $h: f \otimes_{R} n \mapsto\left(f \otimes_{R} I_{N}\right) \circ \rho(n)$. 
This follows by Dubuc's adjoint triangle theorem applied to the diagram

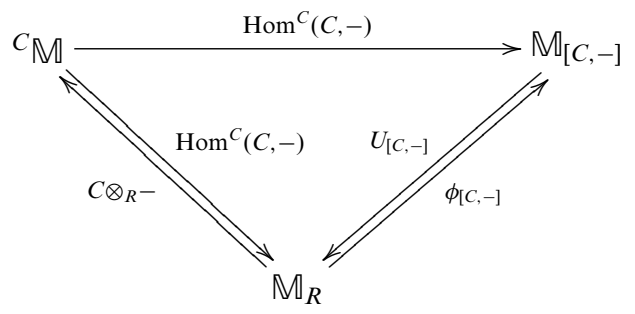

by the existence of the functor in the upper line and the fact that ${ }^{C} \mathbb{M}$ has coequalisers (see [1, Section 4], [3, Appendix] and [6]).

3.10. Equivalence of subcategories. Take any $X \in{ }_{R} \mathbb{M}$. Since $C \otimes_{R}-$ is right adjoint to the forgetful functor, $\operatorname{Hom}^{C}(C,-)$ takes

$$
C \otimes_{R} X \mapsto \operatorname{Hom}^{C}\left(C, C \otimes_{R} X\right) \simeq \operatorname{Hom}_{R}(C, X) .
$$

On the other hand, the functor $C \otimes_{[C,-]}-$ transfers

$$
\operatorname{Hom}_{R}(C, X) \mapsto C \otimes_{[C,-]} \operatorname{Hom}_{R}(C, X) \simeq C \otimes_{R} X .
$$

This shows that the full subcategory of ${ }^{C} \mathbb{M}$, whose objects are of the form $C \otimes_{R} X$, is equivalent to the full subcategory of $\mathbb{M}_{[C,-]}$, with objects $\operatorname{Hom}_{R}(C, X), X \in{ }_{R} \mathbb{M}$ (Kleisli subcategories, e.g. [1]).

Finally we apply the formalism developed to bialgebras and bimodules.

3.11. Bialgebras. An $R$-module $B$ is called an $R$-bialgebra if it is

(i) an algebra $(B, m, e)$ and a coalgebra $(B, \delta, \varepsilon)$,

(ii) the structures are compatible.

Then we know from 2.1 and 2.3 that $\operatorname{Hom}_{R}(B,-)$ is a monad as well as a comonad and certain compatibility conditions hold.

Thus, a bimonad may be defined by properties of two monads $B \otimes_{R}-$ and $\operatorname{Hom}_{R}(B,-)$ or else by the same two functors endowed with comonad structures.

3.12. Bimodules. For an $R$-bialgebra $B$, (mixed) left $B$-bimodules are defined as $R$-modules equipped with a left $B$-module and a left $B$-comodule structure subject to some compatibility conditions. With morphisms respecting the module as well as the comodule structure, they form a category which we denote by ${ }_{B}^{B}$ M. There is a functor

$$
B \otimes_{R}-:{ }_{R} \mathbb{M} \rightarrow{ }_{B}^{B} \mathbb{M}, \quad M \mapsto\left(B \otimes_{R} M, m \otimes I, \delta \otimes I\right),
$$

and $B$ is called a Hopf algebra provided this is an equivalence of categories. The latter can be characterised by the existence of an antipode.

Now, considering the monad and $\operatorname{comonad}[B,-]=\operatorname{Hom}(B,-)$, we can also define bimodules leading to the category $M_{[B,-]}^{[B,-]}$ and the functor

$$
\operatorname{Hom}_{R}(B,-):{ }_{R} \mathbb{M} \rightarrow \mathbb{M}_{[B,-]}^{[B,-]}, \quad M \mapsto\left(\operatorname{Hom}_{R}(B, M), m^{*}, \delta^{*}\right),
$$

which is an equivalence if and only if $B$ is a Hopf algebra. 
These relationships are explained in more detail in [1]. It is shown in [5] and [7] how they can be transferred to bimonads on arbitrary categories.

\section{REFERENCES}

1. G. Böhm, T. Brzeziński and R. Wisbauer, Monads and comonads on module categories, J. Algebra 322 (2009), 1719-1747.

2. T. Brzeziński and R. Wisbauer, Corings and comodules, London Mathematical Society Lecture Note Series 309 (Cambridge University Press, Cambridge, UK, 2003).

3. E. Dubuc, Kan extensions in enriched category theory, Lecture Notes in Mathematics 145 (Springer-Verlag, Berlin, 1970) 398.

4. S. Eilenberg and J. C. Moore, Adjoint functors and triples, Ill. J. Math. 9 (1965), 381-

5. B. Mesablishvili and R. Wisbauer, Bimonads and Hopf monads on categories, J. Ktheory. doi:10.1017/is010001014jkt105 (2010).

6. L. Positselski, Homological algebra of semimodules and semicontramodules, (Birkhauser, Basel, 2010). arXiv:0708.3398v12.

7. R. Wisbauer, Hopf monads on categories, in Noncommutative rings, group rings, diagram algebras and their applications, Contemporary Mathematics, vol. 456 (Jain S. K. et al., Editors) (American Mathematical Society, Providence, RI, 2008), 219-230. 